\title{
Universal quantum gates based on a pair of orthogonal cyclic states: Application to NMR systems
}

\author{
Shi-Liang Zhu ${ }^{1,2, *}$ and Z. D. Wang ${ }^{1,3, \dagger}$ \\ ${ }^{1}$ Department of Physics, University of Hong Kong, Pokfulam Road, Hong Kong, China \\ ${ }^{2}$ Department of Physics, South China Normal University, Guangzhou, China \\ ${ }^{3}$ Department of Material Science and Engineering, University of Science and Technology of China, Hefei, China
}

(Received 28 October 2002; published 28 February 2003)

\begin{abstract}
We propose an experimentally feasible scheme to achieve quantum computation based on a pair of orthogonal cyclic states. In this scheme, quantum gates can be implemented based on the total phase accumulated in cyclic evolutions. In particular, geometric quantum computation may be achieved by eliminating the dynamic phase accumulated in the whole evolution. Therefore, both dynamic and geometric operations for quantum computation are workable in the present theory. Physical implementation of this set of gates is designed for NMR systems. Also interestingly, we show that a set of universal geometric quantum gates in NMR systems may be realized in one cycle by simply choosing specific parameters of the external rotating magnetic fields. In addition, we demonstrate explicitly a multiloop method to remove the dynamic phase in geometric quantum gates.
\end{abstract}

DOI: $10.1103 /$ PhysRevA.67.022319

PACS number(s): 03.67.Lx, 03.65.Vf, 03.67.Pp

\section{INTRODUCTION}

Building a practical quantum computer with a large number of qubits has recently attracted much attention. For realization of a universal quantum computer, there are certain minimum requirements: the storage of quantum information in a set of two-level systems (qubits), the processing of this information using quantum gates, and a mean of final readout [1]. So far, a number of systems has been proposed as potentially viable quantum computer models, including trapped ions [2], cavity quantum electrodynamics [3], nuclear magnetic resonce (NMR) [4], and low-capacitance Josephson juctions [5-8], etc.

An essential requirement in quantum computation is to maintain quantum coherence in a computing system, since the coherent interference pattern between the multitude of superpositions is necessary for taking advantage of quantum parallelism. However, the coupling of a quantum system to its environment leads to the so-called decoherence process, in which encoded quantum information is lost to the environment. The error rates of the individual gate operations should be less than $10^{-4}$ to assure that the quantum computer works fault tolerantly [9]. To accomplish the required precision, the decoherence time of the system has to be much longer than the operation time required for computing. How to suppress the infamous decoherence effects is one main task for quantum computing.

One of schemes to correct the errors caused by decoherence is quantum error-correcting codes [10-12], through which originally encoded information can be recovered by suitable encondings and measurements of qubits. An alternative approach to avoid decoherence has been proposed in Refs. [13,14], where decoherence-free states have been used as qubits. The decoherence-free space is a subspace which is inherently immune to unwanted noise. In addition, evolution

\footnotetext{
*Email address: szhu@hkucc.hku.hk

†Email address: zwang@hkucc.hku.hk
}

of the system must not drive the state out of the decoherencefree space. So far, all of these strategies require extraphysical resources, such as additional manipulations or encoding one logical qubit by several ancillary physical qubits.

Another attractive strategy for fault-tolerant quantum computation is based on a topological idea [15], where gate operations depend only on global features of the control process, and are therefore largely insensitive to local inaccuracies and fluctuations. A significant advance in this direction is made by the so-called geometric quantum computation [16]. In this kind of scheme, a universal set of quantum gates may be realized by pure geometric phases, which depend only on the geometry of the path executed [17-19], and therefore provides a possibility to perform quantum gate operations by an intrinsically fault-tolerant way $[16,20,21]$.

Several basic ideas of adiabatic geometric quantum computation by using NMR [20], superconducting nanocircuits [8], trapped ions [21], or semiconductor nanostructures [22] were proposed. However, the adiabatic evolution appears to be quite special, and thus the nonadiabatic correction on the phase shift may need to be considered in some realistic systems as it may play a significant role in a whole process $[18,23]$. A serious disadvantange of the adiabatic approach is that the evolution time must be much longer than the typical operation time $\tau_{0}$ of the qubit system, while the evolution must be completed within the decoherence time, which leads to an intrinsical time limitation on the operation of quantum gate. Therefore, a generalization to nonadiabatic cases is valuable and important in controlling quantum gates.

Recently, nonadiabatic geometric quantum computation has been proposed theoretically $[24,25]$, and detection of the conditional nonadiabatic geometric phase shifts for quantum gates using NMR is also experimentally reported [26]. Nevertheless a systematic study of this topic, especially the appication to NMR systems, is still highly desirable. In this paper, we propose an experimentally feasible nonadiabatic scheme to achieve a universal set of quantum gates [27] based on a pair of orthogonal cyclic states. In this scheme, quantum gates may be implemented based on either the total 
phase accumulated in the cyclic evolution or the geometric Aharonov-Anandan (AA) phase shift [18] after eliminating the dynamic phase. Therefore, quantum computation implemented by dynamic and geometric operations can be unified in the present theory. In addition, physical implementation of this set of gates is designed in detail for NMR systems, in which the qubits considered are spin-1/2 particles in the presence of a magnetic field rotating uniformly around a fixed axis. Although the Schrödinger equation of this system was solved exactly long time ago [28], and the AA phase was previously obtained explicitly in Refs. [23,29], we here derive all phase shifts explicitly and show that they may be applicable in achieving a universal set of logical gates. Moreover, the nonadiabatic geometric computation may be experimentally achieved just by simply choosing specific controllable parameters, with the cyclic states as a pair of dark states [24].

The paper is organized as follows. In Sec. II, we discuss general aspects of the geometric phase and cyclic evolution, and then present a theory applicable for achieving a universal set of quantum gates based on a pair of orthogonal cyclic states. In Sec. III, the theory is applied to a viable NMR quantum computer. The paper ends with a brief summary.

\section{IMPLEMENTATION OF QUANTUM GATES WITH A PAIR OF ORTHOGONAL CYCLIC STATES}

For universal quantum computation, we need to achieve two kinds of noncommutable single-qubit gates and one nontrivial two-qubit gate [27]. Thus we here consider only twoqubit systems. A general Hamiltonian for two qubits may be expressed as

$$
\hat{H}=-\frac{1}{2} \mu \sum_{i=1}^{3}\left[\sigma_{i}^{(1)} B_{i}^{(1)}(t)+\sigma_{i}^{(2)} B_{i}^{(2)}(t)+J_{i}(t) \sigma_{i}^{(1)} \sigma_{i}^{(2)}\right],
$$

where $\sigma_{i}^{(k)}(k=1,2)$ are the Pauli operators for qubit $k$, $B_{i}^{(k)}(t)$ are local (real or fictitious) magnetic fields acting on $k$ th qubit, and $J_{i}$ represents the strength of the interaction between two qubits.

\section{A. Cyclic evolution and geometric phases}

Before the design of quantum gates, we present first general aspects of the cyclic evolution in a qubit system. A cyclic evolution is referred to as that the state of the system returns to its original state after evolution. Mathematically, a normalized state $|\psi(t)\rangle$ is cyclic in the interval $[0, \tau]$ if and only if

$$
|\psi(\tau)\rangle=e^{i \gamma}|\psi(0)\rangle,
$$

with $\gamma$ being a real number. The total phase $\gamma$ acquired in the evolution would contain both geometric and dynamic components, denoted as $\gamma_{g}$ and $\gamma_{d}$, respectively. Usually, not all states take cyclic evolutions. A sufficient but not necessary condition for cyclic evolution is that the initial state is a nondegenerate energy eigenstate of a cyclic Hamiltonian which changes adiabatically.
We first focus on the cyclic evolution in a qubit system. At this stage, only one term

$$
\hat{H}=-\mu \mathbf{B} \cdot \vec{\sigma} / 2
$$

needs to be considered. Here $\mathbf{B}$ denotes the total magnetic field felt by the qubit, which may include a real external field as well as an effective magnetic field induced by the interaction between different qubits. The Hamiltonian $\hat{H}$ is chosen to go through a cyclic evolution with period $\tau$ in the parameter space $\{\mathbf{B}\}$.

We here present how to calculate all kinds of phase shifts. The dynamic phase may be directly calculated from its definition given by

$$
\gamma_{d}=-\frac{1}{\hbar} \int_{0}^{\tau}\langle\psi(t)|\hat{H}| \psi(t)\rangle d t,
$$

while the geometric part is not easy to derive. We first address a method to calculate adiabatic Berry's phase. By adiabatically changing $\hat{H}$ around a circuit in $\{\mathbf{B}\}$, the eigenstate will accumulate an adiabatic Berry's phase $\gamma_{B}=\mp \Omega_{s} / 2$, where the signs $\mp$ depend on whether the system is in the eigenstate aligned with or against the field, and $\Omega_{s}$ is the solid angle subtended by the magnetic field at the degeneracy $\mathbf{B}=0[17] . \Omega_{s}$ can be derived as

$$
\Omega_{s}=\int_{0}^{\tau_{B_{x}} \partial_{t} B_{y}-B_{y} \partial_{t} B_{x}} \frac{|\mathbf{B}|\left(B_{z}+|\mathbf{B}|\right)}{,}
$$

under the condition of a closed trajectory with $\mathbf{B}(\tau)=\mathbf{B}(0)$ [25].

Since the requirement of the adiabatic evolution could be stringent, a generalization to nonadiabatic case is more desirable. The generalization of adiabatic Berry's phase to a nonadiabatic cyclic evolution was introduced in Ref. [18], where a general geometric phase $\gamma_{g}=\gamma-\gamma_{d}$ is defined as

$$
\gamma_{g}=i \int_{0}^{\tau}\left\langle\widetilde{\psi}(t)\left|\frac{\partial}{\partial t}\right| \widetilde{\psi}(t)\right\rangle
$$

Here $|\widetilde{\psi}(t)\rangle=e^{-i f(t)}|\psi(t)\rangle$ with $f(\tau)-f(0)=\gamma$, leading to $|\widetilde{\psi}(\tau)\rangle=|\widetilde{\psi}(0)\rangle$. The AA phase can be regarded as a geometric phase associated with a closed curve in the projective Hilbert space, and approaches Berry's phase in the adiabatic limit. The AA formulation applies regardless of the Hamiltonian $\hat{H}$ being cyclic or adiabatic; it depends only on the cyclic evolution of the system itself.

Normally, Eq. (5) is not directly used to calculate the geometric phase accumulated in a cyclic evolution. We here present an alternative method to calculate the nonadiabatic geometric phases. This approach is more convenient for qubit systems discussed in this paper. For a spin-1/2 particle in the presence of an arbitrary magnetic field, the nonadiabatic cyclic AA phase is just the solid angle determined by the evolution curve in the projective Hilbert space-a unit sphere $S^{2}$. Any two-component "spin" state $|\psi\rangle$ $=\left[e^{-i \varphi / 2} \cos (\theta / 2), e^{i \varphi / 2} \sin (\theta / 2)\right]^{T}$ may be mapped into a unit 
vector $\mathbf{n}=(\sin \theta \cos \varphi, \sin \theta \sin \varphi, \cos \theta)$ in the projective Hilbert space via the relation $\mathbf{n}=\langle\psi|\vec{\sigma}| \psi\rangle$, where $T$ represents the transposition of matrix. By changing the magnetic field, the AA phase is given by

$$
\gamma_{g}=-\frac{1}{2} \int_{C}(1-\cos \theta) d \varphi
$$

where $C$ is along the actual evolution curve on $S^{2}$, and is determined by the equation

$$
\partial_{t} \mathbf{n}(t)=-\mu \mathbf{B}(t) \times \mathbf{n}(t) / \hbar .
$$

This $\gamma_{g}$ phase recovers Berry's phase in adiabatic evolution [23]. The cyclic evolution implies that $\mathbf{n}(t)$ undergoes a closed path in the projective Hilbert space.

We consider a process, in which a pair of orthogonal states $\left|\psi_{ \pm}\right\rangle$can evolve cyclically starting from $\left|\psi_{ \pm}(0)\right\rangle$. A pair of orthogonal state may be parametrized as

$$
\left|\psi_{+}\right\rangle=\left(\begin{array}{c}
\operatorname{\theta os} \frac{\theta}{2} e^{-i \varphi / 2} \\
\sin \frac{\theta}{2} e^{i \varphi / 2}
\end{array}\right)
$$

and

$$
\left|\psi_{-}\right\rangle=\left(\begin{array}{c}
-\sin \frac{\theta}{2} e^{-i \varphi / 2} \\
\cos \frac{\theta}{2} e^{i \varphi / 2}
\end{array}\right)
$$

Denoting $\mathbf{n}_{ \pm}(t)=\left\langle\psi_{ \pm}(t)|\vec{\sigma}| \psi_{ \pm}(t)\right.$, it is straightforward to find that $\mathbf{n}_{+}(t)=-\mathbf{n}_{-}(t)$ by using Eqs. (8) and (9). For a cyclic evolution, $\left|\psi_{ \pm}(\tau)\right\rangle=e^{i \gamma_{ \pm}}\left|\psi_{ \pm}(0)\right\rangle$. Besides, we have an important relation: $\gamma_{+}=-\gamma_{-}$. This is because the dynamic phase

$$
\gamma_{d+}=-\frac{1}{\hbar} \int_{0}^{\tau} E_{+}(t) d t=-\frac{1}{\hbar} \int_{0}^{\tau}-E_{-}(t) d t=-\gamma_{d-}
$$

with

$$
E_{ \pm}(t)=\left\langle\psi_{ \pm}(t)|H| \psi_{ \pm}\right\rangle=-\mu \mathbf{n}_{ \pm}(t) \cdot \mathbf{B}(t),
$$

and the geometric phase $\gamma_{g}(-\mathbf{n}(0))=-\gamma_{g}(\mathbf{n}(0))$ at any time if the two initial states correspond to $\pm \mathbf{n}(0)$ [23]. By taking into account the cyclic condition for $\left|\psi_{ \pm}\right\rangle$, we finally have

$$
U(\tau)\left|\psi_{ \pm}\right\rangle=\exp ( \pm i \gamma)\left|\psi_{ \pm}\right\rangle,
$$

where $U(\tau)$ is the evolution operator. Hereafter we denote $\gamma, \gamma_{g}$, and $\gamma_{d}$ as the phases for $\left|\psi_{+}\right\rangle$for brevity.

\section{B. Quantum computation}

We now show how to realize a universal set of quantum gates based on either the total phases or the geometric AA phases accumulated in cyclic evolutions.

\section{Quantum logical gates}

A quantum logical gate is a unitary operator $U$ acting on the states of a certain set of qubits, that is, $U$ may be referred to as a quantum gate if $\left|\psi_{\text {out }}\right\rangle=U\left|\psi_{\text {in }}\right\rangle$ with $\left|\psi_{\text {in }}\right\rangle$ being the input state and $\left|\psi_{\text {out }}\right\rangle$ being the output state. The space of all the possible input and output states makes up the Hilbert space of states for the quantum computer. If $\mathcal{H}$ is the Hilbert space of a single qubit, and $\left|\psi_{i}\right\rangle$ is a given basis state for the $i$ th qubit, then a basis vector $|\psi\rangle$ for the states of the quantum register is a tensor product of qubit states $|\psi\rangle=\left|\psi_{1}\right\rangle$ $\otimes\left|\psi_{2}\right\rangle \otimes \cdots \otimes\left|\psi_{n}\right\rangle \in \mathcal{H}^{\otimes n} . \quad U_{N}$ is an $N$-qubit gate when $\left|\psi_{\text {in }}\right\rangle \in \mathcal{H}^{\otimes N}$. Nevertheless, we need not to implement all $U_{l}$ $(1 \leqslant l \leqslant N)$, but only a universal set of gates $U_{u}$. A set of gates $U_{u}$ is called universal if any unitary action $U_{l}$ can be decomposed into a product of successive gates in $U_{u}$. It is shown that two noncommutable one-qubit (single-qubit) gates and one nontrivial two-qubit gate consist of a universal set of gates [27]. This universality is very useful in practice, since it allows us to focus only on how to construct a universal set of gates.

We first construct the single-qubit gates by assuming that a pair of orthogonal states $\left|\psi_{ \pm}\right\rangle$can evolve cyclically. We write an arbitrary input state as $\left|\psi_{i n}\right\rangle=a_{+}\left|\psi_{+}\right\rangle+a_{-}\left|\psi_{-}\right\rangle$ with $a_{ \pm}=\left\langle\psi_{ \pm} \mid \psi\right\rangle$, and express the two cyclic initial states as $\left|\psi_{+}\right\rangle=\cos \chi / 2|0\rangle+\sin \chi / 2|1\rangle$ and $\left|\psi_{-}\right\rangle=-\sin \chi / 2|0\rangle$ $+\cos \chi / 2|1\rangle$, where $|0\rangle$ and $|1\rangle$ constitute the computational basis for the qubit. Using Eq. (10), the output state at time $\tau$ is found to be [25]

$$
\left|\psi_{\text {out }}\right\rangle=U^{s q}(\chi, \gamma)\left|\psi_{\text {in }}\right\rangle,
$$

where

$$
\begin{aligned}
& U^{s q}(\chi, \gamma) \\
& =\left(\begin{array}{ll}
e^{i \gamma} \cos ^{2} \frac{\chi}{2}+e^{-i \gamma} \sin ^{2} \frac{\chi}{2} & i \sin \chi \sin \gamma \\
i \sin \chi \sin \gamma & e^{i \gamma} \sin ^{2} \frac{\chi}{2}+e^{-i \gamma} \cos ^{2} \frac{\chi}{2}
\end{array}\right) .
\end{aligned}
$$

For this gate, there exists a relation

$$
\left[U^{s q}(\chi, \gamma)\right]^{\dagger}=\left[U^{s q}(\chi,-\gamma)\right]
$$

where the adjoint operation $\dagger$ corresponds to transposition and complex conjugation of matrix. Thus the important $\dagger$ operator for a unitary operation $U$ may be experimentally achieved by the operation $U$ with the inverse sign of the phase factor.

It is straightforward to verify that two operations $U^{(1)}\left(\chi_{1}, \gamma_{1}\right)$ and $U^{(2)}\left(\chi_{2}, \gamma_{2}\right)$ are noncommutable as long as

$$
\sin \gamma_{1} \sin \gamma_{2} \sin \left(\chi_{2}-\chi_{1}\right) \neq 0 .
$$

Since two kinds of noncommutable operations constitute a universal set of single-qubit gates, we achieve the universal single-qubit gates by choosing $\chi_{1} \neq \chi_{2}+j \pi$ for any nontrivial phases $\gamma_{1}$ and $\gamma_{2}\left(\gamma_{1,2} \neq j \pi\right)$, where $j$ is an integer. 
For example, the phase-flip gate $U_{1}^{s q}\left(\gamma_{1}\right)=\exp \left(-2 i \gamma_{1}|1\rangle\langle 1|\right)$ (up to an irrelevant overall phase) is accomplished at $\chi$ $=0$; the gate $U_{2}^{s q}\left(\gamma_{2}\right)=\exp \left(i \gamma_{2} \sigma_{x}\right)$ is obtained at $\chi=\pi / 2$, which produces a spin flip (NOT-operation) when $\gamma_{2}=\pi / 2$ and an equal-weight superposition of spin states when $\gamma_{2}$ $=\pi / 4 . U_{1,2}^{s q}$ are two well-known single-qubit gates.

In terms of the computational basis $\{|00\rangle,|01\rangle,|10\rangle,|11\rangle\}$, where the first (second) number represent the state in the controlled (target) qubit, the unitary operator to describe the two-qubit gate is given by $[8,25]$

$$
U^{t q}=\operatorname{diag}\left(U_{\left(\gamma^{0}, \chi^{0}\right)}, U_{\left(\gamma^{1}, \chi^{1}\right)}\right),
$$

under the condition that the control qubit is far away from the resonance condition for the operation of the target qubit. Here $\gamma^{\delta}\left(\chi^{\delta}\right)$ represents the total phase (the cyclic initial state) of the target qubit when the control qubit is in state $\delta(=0,1)$. Following Ref. [27], we find that unitary operator (13) is a nontrivial two-qubit gate if and only if $\gamma^{1} \neq \gamma^{0}$ or $\chi^{1} \neq \chi^{0}(\bmod 2 \pi)$. For example,

$$
U_{\left(\gamma^{0}, \gamma^{1}\right)}^{t q}=\operatorname{diag}\left(e^{i \gamma^{0}}, e^{-i \gamma^{0}}, e^{i \gamma^{1}}, e^{-i \gamma^{1}}\right),
$$

when $\chi^{1}=\chi^{0}=0$; this gate was proposed to be achieved in the adiabatic case in the charge qubit [8]. Combining gate (14) with single-qubit operations we are able to perform a gate described by

$$
\begin{aligned}
U_{C N} & =\left[I \otimes U^{s q}(\pi / 4, \pi / 2)\right] U_{(0, \pi / 2)}^{t q}\left[I \otimes U^{s q}(\pi / 4, \pi / 2)\right]^{\dagger} \\
& =\operatorname{diag}\left(I, i \sigma_{x}\right),
\end{aligned}
$$

with $I$ as a $2 \times 2$ unit matrix. This gate is equivalent to the controlled-NOT (c-NOT, which is defined as $|m\rangle|n\rangle \rightarrow|m\rangle \mid m$ $\oplus n\rangle$, where $\oplus$ denotes the addition modulo 2) gate up to an overall phase factor for the target qubit. On the other hand, $U_{\left(0, \gamma^{1}\right)}^{t q}$ become the controlled-phase [c-PHASE, which is defined as $|m\rangle|n\rangle \rightarrow|m\rangle \exp (i m n \phi)|n\rangle]$ gate by removing a overall phase for the target qubit.

An alternative practical method to achieve the controlledtwo-qubit gate is also available under certain conditions. Denoting the Hamiltonian of the target qubit as $H_{t}$, we may produce $H_{t}=0$ by choosing certain parameters of $H_{t}$ when the controlled qubit is in the state $|0\rangle$, while $H_{t}$ is able to realize a required gate when the controlled qubit is in state $|1\rangle$. Then the gate in this case is given by

$$
U^{t q}=\operatorname{diag}(I, U(\gamma, \chi))
$$

where $\gamma$ is the total phase accumulated in the evolution when the controlled qubit is in state $|1\rangle$. Gate (16) corresponds to gate (13) for $\gamma^{0}=0$ and $\chi_{0}=\pi / 2 . U_{C N}$ in Eq. (15) may be directly derived when $\gamma=\chi=\pi / 2$.

So far, we have demonstrated that all elements of quantum computation may be achievable by using a pair of orthogonal cyclic states.

\section{Geometric quantum gates}

The quantum gates $U$ described in Eqs. (11), (13) [or (16)] may be divided into two categories: one is referred to as a geometric gate if the phase in $U$ is a pure geometric evolution operator $\left(\gamma_{d}=0\right)$ [30], and the other is referred to as a dynamic gate as long as there exists a nonzero phase induced from dynamic origin (i.e., $\gamma_{d} \neq 0$ ). Geometric quantum computation demands that logical gates in computing are realized by using geometric phase shifts, so that it may have the built-in fault-tolerant advantage due to the fact that the geometric phases depend only on some global geometric features.

A key point in geometric quantum computation is to remove the dynamic phase. We here address two methods $[8,20,21,24,25]$. A simpler and also practical one is to choose some specific external parameters such that the dynamic phases of the pair of cyclic states accumulated in the whole evolution may be eliminated. Interestingly, with this method the corresponding cyclic states in NMR systems are dark states (the eigenstate with the zero-energy eigenvalue), and thus no dynamic phase is involved. The dark state method was proposed for geometric quantum computation with trapped ions [21], and then described in NMR systems [24]. The other is referred to as a two-loop method: let the evolution be dragged by $\hat{H}$ along two closed loops, with one being in $t \in[0, \tau]$ and the other in $t \in\left[\tau, \tau+\tau^{\prime}\right]$. The dynamic phases accumulated in the two loops may be canceled, while the AA phases will add.

\section{APPLICATION TO NMR SYSTEMS}

So far, we have proposed a general scheme to achieve a universal set of quantum gates based on a pair of orthogonal cyclic states. It is important to further consider implementing this scheme with real physical systems. Here, we illustrate this implementation using NMR systems $[4,20]$. Nevertheless, it is worth pointing out that, in principle, the above theory may be applicable to other systems which are potentially viable quantum computer models.

For NMR systems, the magnetic field in Eq. (1) or (2) in a rotating magnetic field is given by

$$
\mathbf{B}(t)=\left(B_{0} \cos \omega t, B_{0} \sin \omega t, B_{1}\right),
$$

where $B_{0,1}$ and $\omega$ are constants. The qubit state $|\psi(t)\rangle$ is described by the Schrödinger equation

$$
i \hbar \frac{\partial}{\partial t}|\psi(t)\rangle=H|\psi(t)\rangle
$$

where the Hamiltonian for a single qubit is given by

$$
H=\frac{1}{2}\left(\omega_{0} \sigma_{x} \cos \omega t+\omega_{0} \sigma_{y} \sin \omega t+\omega_{1} \sigma_{z}\right)
$$

with $\omega_{i}=-g \mu B_{i} / \hbar(i=0,1)$ and $g$ being the gyromagnetic ratio. The Schrödinger equation with Hamiltonian (19) can be solved analytically $[23,28]$. In terms of explicit form of the solution $\mathbf{n}(\chi, \omega t)$ represented in Ref. [23], it is found that 
a pair of orthogonal initial states $\left|\psi_{ \pm}\right\rangle$with $\chi$ $=\arctan \left[\omega_{0} /\left(\omega_{1}-\omega\right)\right]$ take cyclic evolutions with the period $\tau=2 \pi / \omega[24]$, and the evolution paths are the curves on a Bloch sphere swept by unit vectors $\pm \mathbf{n}(\chi, \omega t)$. Therefore, we may use this pair of $\left|\psi_{ \pm}\right\rangle$to achieve single-qubit gates described in Eq. (11), where the corresponding phases for one cycle are given by

$$
\begin{gathered}
\gamma_{g}=-\pi\left(1-\frac{\omega_{1}-\omega}{\Omega}\right), \\
\gamma_{d}=-\pi \frac{\omega_{0}^{2}+\omega_{1}\left(\omega_{1}-\omega\right)}{\omega \Omega}, \\
\gamma=-\pi(1+\Omega / \omega),
\end{gathered}
$$

with $\Omega=\sqrt{\omega_{0}^{2}+\left(\omega_{1}-\omega\right)^{2}}$. In the derivation of the dynamic phase, $E_{+}(t)=\left[\omega_{1} \cos \chi+\omega_{0} \sin \chi\right] \hbar / 2$ is used. We may choose any two processes with different values $\left\{\omega_{0}, \omega_{1}, \omega\right\}$ satisfying Eq. (12) to accomplish two noncommutable qubit gates.

A similar method may be employed to achieve the twoqubit operation. The spin-spin interaction in NMR is very well approximated by

$$
H_{I}=J \sigma_{z}^{1} \sigma_{z}^{2} / 2
$$

The state of control qubit is (almost) not affected by any operation of the target qubit if $\omega_{1}^{t}$ of the target qubit is chosen to be significantly different from $\omega_{1}^{c}$ of the control qubit. We may prove that the initial states $\left|\psi_{ \pm}\right\rangle$described by $\chi^{\delta}$ $=\arctan \left[\omega_{0} /\left(\omega_{1}^{\delta}-\omega\right)\right]$ are a pair of orthogonal cyclic states, and may be used to achieve a two-qubit gate described by Eq. (13). Here $\omega_{1}^{\delta}=\omega_{1}+(2 \delta-1) J, \omega, \omega_{0}$, and $\omega_{1}$ are parameters for the target qubit (the superscript " $t$ " is omitted for brevity). The corresponding phases for one cycle are given by

$$
\begin{gathered}
\gamma_{g}^{\delta}=-\pi\left(1-\frac{\omega_{1}^{\delta}-\omega}{\Omega^{\delta}}\right), \\
\gamma_{d}^{\delta}=-\pi \frac{\omega_{0}^{2}+\omega_{1}^{\delta}\left(\omega_{1}^{\delta}-\omega\right)}{\omega \Omega^{\delta}}, \\
\gamma^{\delta}=-\pi\left(1+\Omega^{\delta} / \omega\right),
\end{gathered}
$$

with $\Omega^{\delta}=\sqrt{\omega_{0}^{2}+\left(\omega_{1}^{\delta}-\omega\right)^{2}}$. It is seen from Eq. (25) that the gate described by Eq. (16) may be accomplished by choosing the following special parameters,

$$
\omega=\omega_{0}=\omega_{1}-J
$$

It is worth pointing out that we may achieve the nonadiabatic geometric gates by choosing some specific parameters, with which $\gamma_{d}=0$ in the whole process. It is direct to verify that the dynamic phase in Eq. (21) is zero under the following condition,

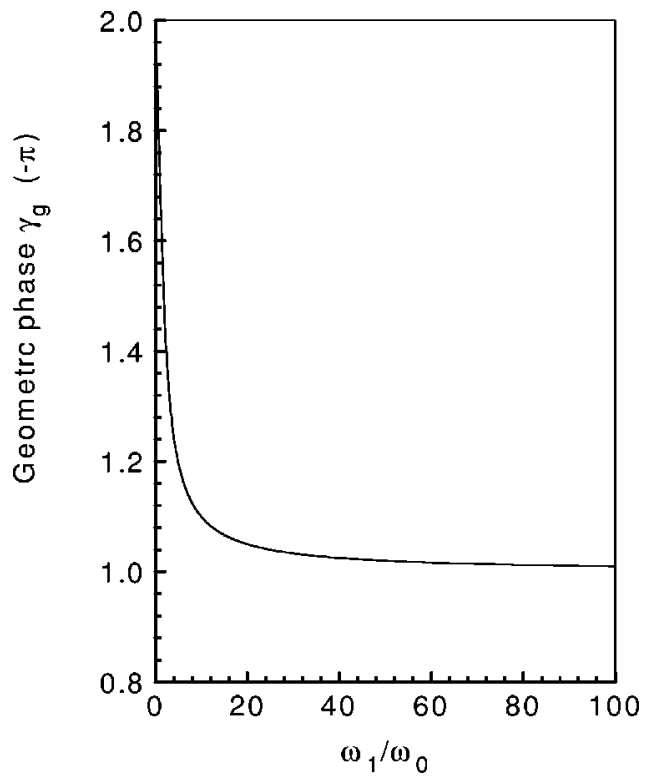

FIG. 1. The geometric phase $\gamma_{g}$ versus the ratio $\omega_{1} / \omega_{0}$.

$$
\omega=\frac{\omega_{0}^{2}+\omega_{1}^{2}}{\omega_{1}} .
$$

Thus the single-qubit gates with the parameters satisfying Eq. (26) are geometric quantum gates with geometric phase shift $\gamma_{g}=-\pi\left(1+\omega_{0} / \sqrt{\omega_{0}^{2}+\omega_{1}^{2}}\right)$. The geometric phases $\gamma_{g}$ versus the ratio $\omega_{1} / \omega_{0}$ are plotted in Fig. 1 . It is seen that the nontrivial phases required for two universal single-qubit gates may be simply realized by any two processes with different values of $\omega_{1} / \omega_{0}$ (except for zero or infinite).

Besides, the geometric two-qubit gates are realized whenever

$$
\begin{gathered}
\omega=2 \omega_{1}, \\
\omega_{1}^{2}=\omega_{0}^{2}+J^{2} .
\end{gathered}
$$

Correspondingly, the conditional geometric phases are given by $\gamma_{g}^{\delta}=-\pi\left(1+\sqrt{\omega_{1}^{\delta} / 2 \omega}\right)$. Figure 2 shows the conditional phases $\gamma_{g}^{\delta}$ versus the ratio $\omega_{1} / J$. It is evident that the nontrivial phases $\left(\gamma_{g}^{0} \neq \gamma_{g}^{1}\right.$ in $\left.\bmod 2 \pi\right)$ required for two-qubit gates may be achieved for $0<\omega_{1} / J<\infty$.

It is worth pointing out that the constraint described by Eq. (26) [or Eqs. (27) and (28)] is equivalent to the condition that the instantaneous dynamic phase for the wave function in single qubit (or the target qubit) is always zero [24], namely, the states $\left|\psi_{ \pm}\right\rangle$used here are the dark states.

The advantage of the above nonadiabatic gates is that $\omega$ is of the same order of magnitude as $\omega_{0}$ or $\omega_{1}$. This implies that the speed of geometric quantum gate is comparable with that of the dynamic quantum gate. In contrast, the speed of quantum gate based on adiabatic Berry's phase is much lower than that of gate using dynamic phase, since the adiabatic condition requires that both $\omega_{0}$ and $\omega_{1}$ should be much larger than $\omega$. 


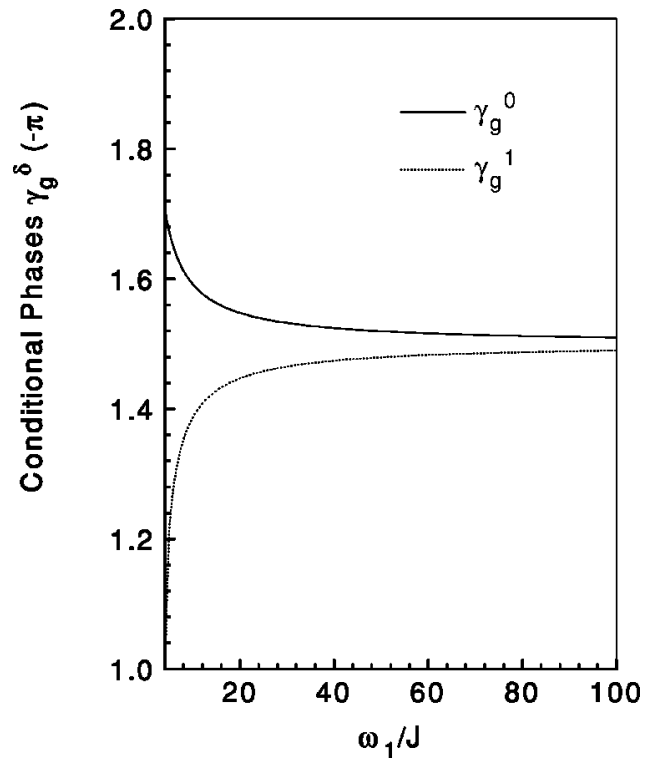

FIG. 2. The conditional phases $\gamma_{g}^{\delta}$ versus the ratio $\omega_{1} / J$.

Note that, the geometric gates $U_{1,2}^{s q}$ may not be practical by directly using the field $\mathbf{B}$ in Eq. (17) as the corresponding geometric phase in Eq. (20) is determined by the angle $\chi$. For example, Eq. (20) can be rewritten as $\gamma_{g}=-\pi(1$ $-\cos \chi)$; thus $\gamma_{g}=0(-\pi)$ as $\chi=0(\pi / 2)$. This problem can be solved by rotating the field. It will be seen below that the parameter $\chi$ for the initial cyclic state may be changed by rotating the symmetric axis of field (17), while the phases in Eqs. (20), (21), and (22) are invariant.

We introduce a rotation operator $\mathbf{R}\left(\hat{y}, \chi^{\prime}-\chi\right)$ that represents the rotation of angle $\chi^{\prime}-\chi$ around the $\hat{y}$ axis, that is,

$$
\mathbf{R}^{(2)}\left(\hat{y}, \chi^{\prime}-\chi\right)=\exp \left[-i\left(\chi^{\prime}-\chi\right) \sigma_{y}\right]
$$

in the $\mathrm{SU}(2)$ representation, and

$$
\mathbf{R}^{(3)}\left(\hat{y}, \chi^{\prime}-\chi\right)=\exp \left[-i\left(\chi^{\prime}-\chi\right) \tau_{2}\right]
$$

with

$$
\tau_{2}=\left(\begin{array}{lll}
0 & 0 & i \\
0 & 0 & 0 \\
-i & 0 & 0
\end{array}\right)
$$

in the $\mathrm{SO}(3)$ representation. Assuming the required angle is $\chi^{\prime}$ in Eq. (11), we may apply a magnetic field $\mathbf{B}^{\prime}=\mathbf{R}^{(3)}\left(\hat{y}, \chi^{\prime}-\chi\right) \mathbf{B}$, then the solution of the cyclic states are $\quad \mathbf{n}_{ \pm}^{\prime}=\mathbf{R}^{(3)}\left(\hat{y}, \chi^{\prime}-\chi\right)\left[ \pm \mathbf{n}_{ \pm}(\chi, \omega t)\right] \quad\left[\left|\psi_{ \pm}\right\rangle=\mathbf{R}^{(2)}\left(\hat{y}, \chi^{\prime}\right.\right.$ $\left.-\chi)\left|\psi_{ \pm}\right\rangle\right]$because of the spherical symmetry of the system. Thus $\chi$ may change to any required $\chi^{\prime}$ for implementation of the quantum gate, with the geometric phase being unchanged, because the area swept by $\mathbf{n}^{\prime}$ is the same as that by n. On the other hand, we have

$$
\begin{aligned}
E_{ \pm}^{\prime}(t) & =-\mu \mathbf{n}_{ \pm}^{\prime}(t) \cdot \mathbf{B}^{\prime}(t) \\
& =-\mu\left[\mathbf{R}^{(3)}\left(\hat{y}, \chi^{\prime}-\chi\right) \mathbf{n}_{ \pm}(t)\right] \cdot\left[\mathbf{R}^{(3)}\left(\hat{y}, \chi^{\prime}-\chi\right) \mathbf{B}(t)\right] \\
& =E_{ \pm}(t) .
\end{aligned}
$$

Therefore, we have proven that the invariant of all phases with respect to the rotation of the symmetric axis of the field in Eq. (17). We conclude that $\gamma(\chi)$ in gate (11) is determined by the values of $\left\{\omega_{0}, \omega_{1}, \omega\right\}$ (the symmetric axis of the magnetic field). For example, if the magnetic field is $\mathbf{B}^{\prime}$ for $\chi^{\prime}=0(\pi / 2)$, we may achieve the geometric gate $U_{1}$ $\left(U_{2}\right)$ with $\gamma_{1,2}=-\pi(1-\cos \chi)$. It is worth pointing out that the above method to control $\chi$ and $\gamma_{g}$ separately in quantum gates is also feasible in nongeometric gates.

We turn to address how to remove the dynamic phases in a multiloop nonadiabatic evolution. The possible generalization of a multiloop method from the adiabatic evolution $[8,20]$ to nonadiabatic case was mentioned in Refs. [24,25]. We here wish to demonstrate explicitly one removal procedure of the dynamic phase.

Let us first choose the magnetic fields in two loops as

Loop 1.

$$
B=\left(B_{0} \cos \omega t, B_{0} \sin \omega t, B_{1}\right), \quad t \in[0, \tau) .
$$

Loop 2.

$$
\begin{gathered}
B^{\prime}=\mathbf{R}^{(3)}\left(\hat{y}, \alpha^{\prime}-\alpha\right)\left(-B_{0}^{\prime} \cos \omega t,-B_{0}^{\prime} \sin \omega t,-B_{1}^{\prime}\right), \\
t \in[\tau, 2 \tau],
\end{gathered}
$$

where $\quad \tau=2 \pi / \omega, \quad \alpha=\arctan \left[\omega_{0} /\left(\omega_{1}-\omega\right)\right], \quad$ and $\quad \alpha^{\prime}$ $=\arctan \left[\omega_{0}^{\prime} /\left(\omega_{1}^{\prime}+\omega\right)\right]$ with $\omega_{i}^{\prime}=-g \mu B_{i}^{\prime} / \hbar \quad(i=0,1)$. As shown before, a pair of orthogonal initial states $\left|\psi_{ \pm}\right\rangle\left(\left|\psi_{ \pm}^{\prime}\right\rangle\right)$ with $\chi=\alpha\left(\chi^{\prime}=\alpha^{\prime}\right)$ take cyclic evolutions during the loop one (two). The rotation $\mathbf{R}^{(3)}\left(\hat{y}, \alpha^{\prime}-\alpha\right)$ in Eq. (33) ensures that the cyclic initial states in the two loops are the same at the time $t=2 \pi / \omega$ [31]. Therefore, the gate described by the two loops is given by $U=U\left(\chi, \gamma^{(1)}+\gamma^{(2)}\right)$, where $\gamma^{(1)}$ $\left(\gamma^{(2)}\right)$ is the total phase accumulated in the loop one (two). Denoting $\gamma_{d}^{(l)}(l=1,2)$ and $\gamma_{g}^{(l)}$ the dynamic phases and geometric phases accumulated in the loop $l$, respectively, we now illustrate that there exist processes satisfying

$$
\begin{gathered}
\gamma_{g}^{(1)}+\gamma_{g}^{(2)}=-\Gamma \pi, \\
\gamma_{d}^{(1)}+\gamma_{d}^{(2)}=0,
\end{gathered}
$$

where $-\Gamma \pi$ is a nontrivial geometric phase which we intend to realize in geometric quantum gates. Then the magnetic fields should satisfy the following equations:

$$
\begin{gathered}
\frac{\omega_{1}-\omega}{\Omega}+\frac{\omega_{1}^{\prime}+\omega}{\Omega^{\prime}}=2-\Gamma, \\
\frac{\omega_{0}^{2}+\omega_{1}^{2}-\omega \omega_{1}}{\omega \Omega}=\frac{\left(\omega_{0}^{\prime}\right)^{2}+\left(\omega_{1}^{\prime}\right)^{2}+\omega \omega_{1}^{\prime}}{\omega \Omega^{\prime}},
\end{gathered}
$$

where $\Omega=\sqrt{\omega_{0}^{2}+\left(\omega_{1}-\omega\right)^{2}}$ and $\Omega^{\prime}=\sqrt{\left(\omega_{0}^{\prime}\right)^{2}+\left(\omega_{1}^{\prime}+\omega\right)^{2}}$. As for the required $\Gamma$, it is possible that there exist many solutions, since there are five unknown variables in two equations. For example, we numerically calculate the solu- 
tions for $\Gamma=1 / 2$. For simplicity, we set $\omega_{1}^{\prime}=\omega_{1}$ as the unit, and find that if $\left\{\omega, \omega_{0}, \omega_{0}^{\prime}\right\}\left(0<\omega<\omega_{1}\right)$ satisfy the equations given by

$$
\begin{gathered}
\omega+1.13389 \omega_{0}=0.99998, \\
\omega+1.07091 \omega_{0}-0.06299 \omega_{0}^{\prime}=0.88889,
\end{gathered}
$$

which describe a straight line(segment) in the threedimensional space, the geometric phase accumulated in the whole two-loop evolution is just what we required, with the total dynamic phase being zero.

The multiloop method to remove dynamic phase is also feasible for two-qubit geometric quantum gates. We choose the magnetic fields on the target qubit in two loops as

Loop 1.

$$
B=\left(B_{0} \cos \omega t, B_{0} \sin \omega t, B_{1}\right), \quad t \in[0, \tau),
$$

Loop 2.

$$
\begin{gathered}
B^{\prime}=\mathbf{R}^{(3)}(\hat{y}, \eta)\left(-B_{0}^{\prime} \cos \omega^{\prime} t,-B_{0}^{\prime} \sin \omega^{\prime} t,-B_{1}^{\prime}\right), \\
t \in\left[\tau, \tau+\tau^{\prime}\right],
\end{gathered}
$$

where $\tau^{\prime}=2 \pi / \omega^{\prime}$. The angle

$$
\eta=\arctan \left[\omega_{0} /\left(\omega_{1}^{\delta}-\omega\right)\right]-\arctan \left[\omega_{0}^{\prime} /\left(\omega_{1}^{\prime} \delta+\omega^{\prime}\right)\right]
$$

should be independent on the state $\delta$ in the control qubit. To guarantee that the interaction between qubits is still determined by the original initial state $\delta$ of the control qubit, the control qubit should be rotated by $\mathbf{R}^{(3)}(\hat{y}, \eta)$ at time $t=\tau$ [The state of the controlled qubit is unchanged if a rotation $\mathbf{R}^{(3)}(\hat{y},-\eta)$ is also applied at time $\left.\tau+\tau^{\prime}\right]$. Correspondingly, the $\delta$-independent constraint described by Eq. (38) can be rewritten as

$$
\frac{\omega_{0}}{\left(\omega_{1}-\omega\right)^{2}-J^{2}}=\frac{\omega_{0}^{\prime}}{\left(\omega_{1}^{\prime}+\omega^{\prime}\right)^{2}-J^{2}} .
$$

On the other hand, the condition under which there exist processes with zero dynamic phase is

$$
\frac{\omega_{0}^{2}+\omega_{1}^{\delta}\left(\omega_{1}^{\delta}-\omega\right)}{\omega \Omega^{\delta}}=\frac{\left(\omega_{0}^{\prime}\right)^{2}+\omega_{1}^{\prime} \delta\left(\omega_{1}^{\prime} \delta+\omega^{\prime}\right)}{\omega^{\prime} \Omega^{\prime} \delta},
$$

where $\quad \Omega^{\delta}=\sqrt{\omega_{0}^{2}+\left(\omega_{1}^{\delta}-\omega\right)^{2}} \quad$ and $\quad \Omega^{\prime \delta}$ $=\sqrt{\left(\omega_{0}^{\prime}\right)^{2}+\left(\omega_{1}^{\prime \delta}+\omega^{\prime}\right)^{2}}$. Note that the geometric phases are nontrivial $\left(\gamma_{g}^{1} \neq \gamma_{g}^{0}\right.$ in $\left.\bmod 2 \pi\right)$, and thus can be applicable in geometric quantum computation.

The magnetic fields, which satisfy Eqs. (39) and (40) in loop two, as a function of $\omega$ are plotted in Fig. 3(a), where $\omega_{0}=\omega_{1}=5.0$ with $J$ as the unit. We may numerically calculate the three unknown variables $\left\{\omega^{\prime}, \omega_{0}^{\prime}, \omega_{1}^{\prime}\right\}$ in three equations described by Eqs. (39) and (40). Then the conditional geometric phases may be obtained from equations $\gamma_{g}^{\delta}=$ $-\Gamma^{\delta} \pi$ with
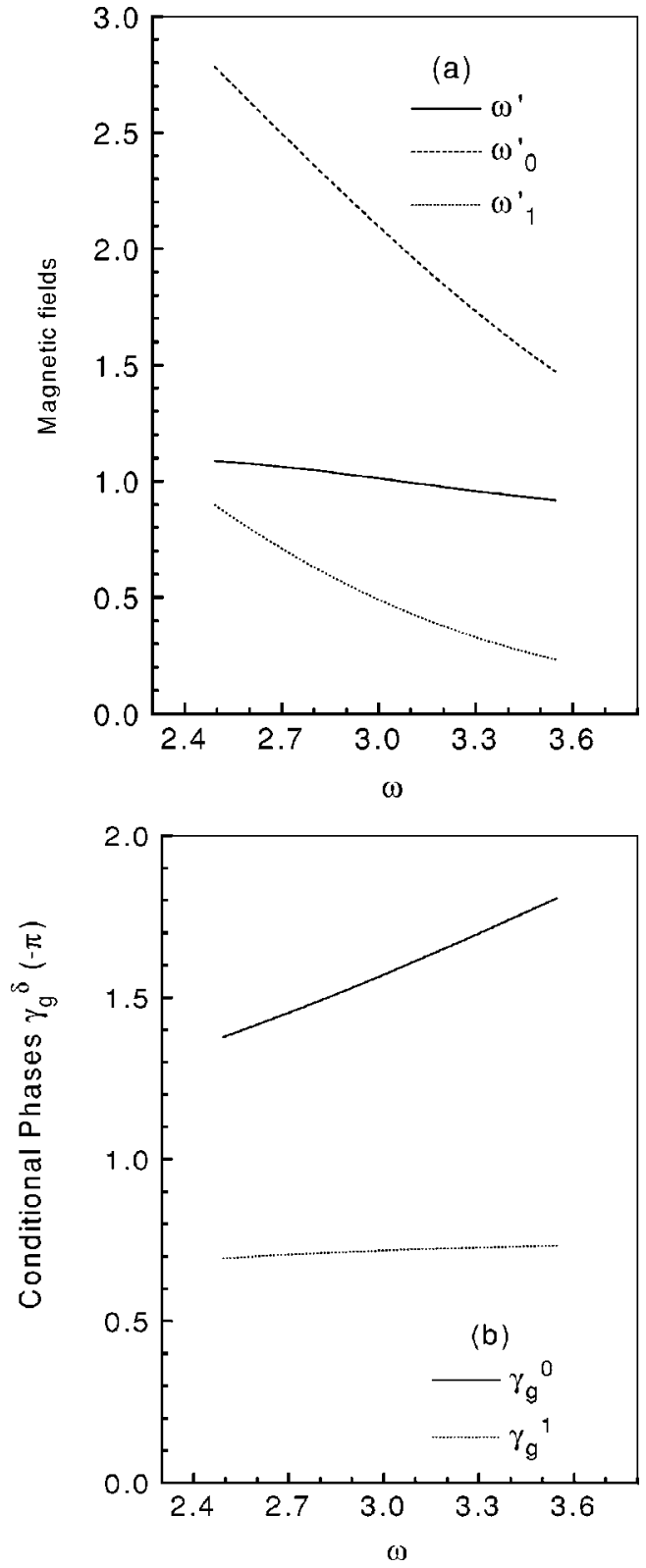

FIG. 3. (a) The magnetic fields required in loop two versus $\omega$. (b) The conditional geometric phases $\gamma_{g}^{\delta}$ versus $\omega$.

$$
\Gamma^{\delta}=2-\frac{\omega_{1}^{\delta}-\omega}{\Omega^{\delta}}-\frac{\omega_{1}^{\prime \delta}+\omega^{\prime}}{\Omega^{\prime} \delta} .
$$

The corresponding conditional phases $\gamma_{g}^{\delta}$ for $\omega_{0}=\omega_{1}=5.0$ as a function of $\omega$ are plotted in Fig. 3(b). It is seen that the nontrivial phases $\gamma_{g}^{1} \neq \gamma_{g}^{0}$ may be realized by appropriately choosing the values of $\left\{\omega, \omega_{0}, \omega_{1}\right\}$ and $\left\{\omega^{\prime}, \omega_{0}^{\prime}, \omega_{1}^{\prime}\right\}$. As a consequence the nontrivial two-qubit geometric quantum gate may be achieved.

\section{CONCLUSIONS AND DISCUSSIONS}

An experimentally feasible scheme based on a pair of orthogonal cyclic states has been proposed to accomplish a 
universal set of quantum logical gates, in which quantum computation implemented by both dynamic and geometric operations can work, i.e., quantum gates in this scheme may be implemented by the total phases accumulated in the cyclic evolution, and the geometric quantum computation can be achieved by eliminating the dynamic phase. Furthermore, the geometric phase shift used is the cyclic AA phase, which can be nonadiabatic. It is possible that the gates achieved here can handle arbitrary quantum computation without the intrinsic limitation on operation time. Therefore, the nonadiabatic method proposed here may allow us to physically implement (geometric) quantum computation even for systems with very short decoherence time, which could be especially useful for solid-state implementations of scalable quantum computers.

We here discuss briefly the errors induced by random noises in geometric quantum computation. Random noises may lead to two kinds of errors. One is that the path may not be exactly closed at the end of the gate operation, leading to the noncyclic corrections. The other is that the evolution path may fluctuate around the ideal path with known cone angle. The noncyclic corrections could be negligible at least when the first-order corrections from random noises are taken into account, as indicated in Ref. [32]. On the other hand, as that in the adiabatic cyclic geometric scheme, the present scheme is also robust to the second type of errors as the area enclosed by the evolution path (geometric phase) is insensitive to the random fluctuation.
Finally, we wish to make a few remarks on experimental implementation of geometric quantum computation. The simplest geometric quantum computation should experimentally complete the following three steps one by one: (i) detection of the (conditional) geometric phase shifts in qubit systems; (ii) implementation of a universal set of geometric quantum logic gates, particularly the implementation of a conditional gate. (iii) illustration of a simple algorithm by pure geometric quantum gates, such as Deutsch's problem, Grover's search algorithm, or Shor's factorization algorithm, etc. Two recent exciting experiments reported that the conditional geometric phase shifts for quantum logical gates using NMR were detected in adiabatic [20] and nonadiabatic [26] regions. However, a universal set of gates as well as a simple quantum algorithm experimented by (adiabatic or nonadiabatic) geometric phases are still awaited.

\section{ACKNOWLEDGMENTS}

We are very grateful to Dr. L. M. Duan, Dr. J. W. Pan, and Dr. X. B. Wang for valuable discussions, and Dr. P. Zanardi for pointing out Ref. [26]. This work was supported by the RGC grant of Hong Kong under Grants Nos. HKU7118/00P and HKU7114/02P, and a CRCG grant at the HKU. S.L.Z. was supported in part by SRF for ROCS, SEM, the NSF of Guangdong Province under Grant No. 021088, and the NNSF of China under Grant No. 10204008.
[1] D.P. DiVincenzo, Fortschr. Phys. 48, 771 (2000).

[2] J.I. Cirac and P. Zoller, Phys. Rev. Lett. 74, 4091 (1995).

[3] T. Pellizzari, S.A. Gardiner, J.I. Cirac, and P. Zoller, Phys. Rev. Lett. 75, 3788 (1995); Q.A. Turchette, C.J. Hood, W. Lange, H. Mabuchi, and H.J. Kimble, ibid. 75, 4710 (1995).

[4] D.G. Cory, A.F. Fahmy, and T.F. Havel, in Proceedings of PhysComp'96, edited by T. Toffoli, M. Biafore, and J. Leao (New England Complex Systems Institute, Cambridge, 1996), p. 87; N.A. Gershenfeld and I.L. Chuang, Science 275, 350 (1997); J.A. Jones and M. Mosca, J. Chem. Phys. 109, 1648 (1998).

[5] A. Shnirman, G. Schön, and Z. Hermon, Phys. Rev. Lett. 79, 2371 (1997); D.V. Averin, Solid State Commun. 105, 659 (1998).

[6] Y. Makhlin, G. Schön, and A. Shnirman, Nature (London) 398, 305 (1999); Rev. Mod. Phys. 73, 357 (2001).

[7] Y. Nakamura, Yu.A. Pashkin, and J.S. Tsai, Nature (London) 398, 786 (1999); Physica B 280, 405 (2000).

[8] G. Falci, R. Fazio, G.M. Palma, J. Siewert, and V. Vedral, Nature (London) 407, 355 (2000).

[9] J. Preskill, Proc. R. Soc. London, Ser. A 454, 385 (1998); A.M. Steane, Nature (London) 399, 124 (1999).

[10] P.W. Shor, Phys. Rev. A 52, R2493 (1995).

[11] C.H. Bennett, D.P. DiVincenzo, J.A. Smolin, and W.K. Wootters, Phys. Rev. A 54, 3824 (1996).

[12] A.M. Steane, Phys. Rev. Lett. 77, 793 (1996).

[13] L.M. Duan and G.C. Guo, Phys. Rev. Lett. 79, 1953 (1997); Phys. Rev. A 58, 3491 (1998).
[14] P. Zanardi and M. Rasetti, Phys. Rev. Lett. 79, 3306 (1997).

[15] A.Y. Kitaev, e-print quant-ph/9707021.

[16] P. Zanardi and M. Rasetti, Phys. Lett. A 264, 94 (1999).

[17] M.V. Berry, Proc. R. Soc. London, Ser. A 392, 45 (1984).

[18] Y. Aharonov and J. Anandan, Phys. Rev. Lett. 58, 1593 (1987).

[19] H.Z. Li, Global Properties of Simple Physical Systems (Shanghai Scientific and Technical Publishers, Shanghai, 1998).

[20] J.A. Jones, V. Vedral, A. Ekert, and G. Castagnoli, Nature (London) 403, 869 (2000).

[21] L.M. Duan, J.I. Cirac, and P. Zoller, Science 292, 1695 (2001).

[22] P. Solinas, P. Zanardi, N. Zanghi, and F. Rossi, e-print quant-ph/0207019.

[23] S.L. Zhu and Z.D. Wang, Phys. Rev. Lett. 85, 1076 (2000); S.L. Zhu, Z.D. Wang, and Y.D. Zhang, Phys. Rev. B 61, 1142 (2000).

[24] X.B. Wang and M. Keiji, Phys. Rev. Lett. 87, 097901 (2001); 88, 179901(E) (2002); Phys. Rev. B 65, 172508 (2002); J. Phys. A 34, L631 (2001).

[25] S.L. Zhu and Z.D. Wang, Phys. Rev. Lett. 89, 097902 (2002); ibid. 89, 289901(E) (2002); Phys. Rev. A 66, 042322 (2002).

[26] J. Du, M. Shi, J. Wu, X. Zhou, and R. Han, e-print quant-ph/0207022.

[27] D. Deutsch, A. Barenco, and A. Ekert, Proc. R. Soc. London, Ser. A 449, 669 (1995); S. Lloyd, Phys. Rev. Lett. 75, 346 (1995).

[28] I.I. Rabi, N.F. Ramsey, and J. Schwinger, Rev. Mod. Phys. 26, 167 (1954). 
[29] Q.G. Lin, J. Phys. A 35, 377 (2002); ibid. 34, 1903 (2001); G. Ni, S. Chen, and Y. Shen, Phys. Lett. A 197, 100 (1995).

[30] Here the dynamic phase accumulated in the gate operation is possibly nonzero when an input state is a superposition of $\left|\psi_{ \pm}\right\rangle$. However, it is possible to define a more strictly geometric gate $U$ where the phase accumulated is a pure geometric phase for any input state. The latter gates have not been stud- ied here.

[31] This is a general approach. Actually, $\mathbf{R}^{(3)}\left(\hat{y}, \alpha^{\prime}-\alpha\right)$ in Eq. (33) is not necessary in some cases. For example, another constraint $\alpha^{\prime}-\alpha=0$ may be simultaneously satisfied by appropriate choosing parameters in the example addressed below.

[32] G.M. Palma and G.D. Chiara (unpublished), available at http:// agenda.ictp.trieste.it 